\title{
Spin-Polarized and Normal Hopping Magnetoresistance in Heavily Doped Silicon
}

\author{
A. Fedotov ${ }^{a *}$, S. Prischepa ${ }^{b}$, A. DAnilyuk $^{b}$, I. Svito $^{a}$ And P. Zukowski ${ }^{c}$ \\ ${ }^{a}$ Belarusian State University, Minsk, Belarus \\ ${ }^{b}$ Belarusian State University of Informatics and Radioelectronics, Minsk, Belarus \\ ${ }^{c}$ Lublin University of Technology, Lublin, Poland
}

\begin{abstract}
Investigation of electrical resistivity $\rho$ and magnetoresistance in single crystalline $n$-type silicon heavily doped with antimony in the temperature range $\Delta T=5-300 \mathrm{~K}$ and at the magnetic inductance $B$ up to $8 \mathrm{~T}$ was performed. It was established that, for the temperature range $\Delta T=25-300 \mathrm{~K}$ the conductivity is of activation type, while for $\Delta T=5-25 \mathrm{~K}$ it is of variable range hopping and is described by the Mott law. Parameters of the Mott hopping were calculated. It was shown that, to explain the experimental data, the spin polarized hopping via the occupied states has to be taken into account. The obtained parameters revealed that for the low temperature range $\Delta T=5-11 \mathrm{~K}$ the spin polarized hopping dominates, while for $\Delta T=11-20 \mathrm{~K}$ the spin polarized transport is accompanied by the wave function contraction.
\end{abstract}

DOI: $10.12693 /$ APhysPolA.125.1271

PACS: 61.72.uf, 72.20.My, 75.76. $+\mathrm{j}$

\section{Introduction}

The behavior of the temperature $T$ and magnetic field $B$ dependences of conductivity $\sigma$ in heavily doped semiconductors while approaching the insulator-metal transition (IMT) was the subject of numerous experimental and theoretical investigations over the last 20 years. IMT occurs when the Mott criterion $N_{\mathrm{c}}^{1 / 3} a_{\mathrm{B}} \approx 0.25$ is satisfied ( $N_{\mathrm{C}}$ being a critical concentration of the IMT and $a_{\mathrm{B}}$ the effective Bohr radius of an isolated defect center) [1]. This criterion was confirmed in various experiments [2]. However, some ambiguity in understanding of both $\sigma$ and magnetoresistance (MR) mechanisms still remains when semiconductor is close to the critical concentration of the localized centers and the competition between variable range hopping (VRH), weak localization and standard metallic conductivity occurs.

This paper describes temperature dependences of $\mathrm{dc}$ conductivity $\sigma(T)$, the Hall effect and $I-V$ characteristics of single crystalline $n$-type silicon heavily doped with antimony with concentration $N_{\mathrm{Sb}} \approx 1 \times 10^{18} \mathrm{~cm}^{-3}$ (estimated by the Hall measurements) which is below the $N_{\text {c }}$ and $N_{\text {Sb }}$ values in Si studied in [3]. Rectangular samples were covered with 6 indium stripes as electric probes ( 2 currents and 4 potentials) using ultrasonic soldering. Samples were inserted into the computerized cryogen-free measuring system CFMS (Cryogenic Ltd., London) with superconducting magnet. The CFMS allowed performing measurements in the temperature range $\Delta T$ between $5 \mathrm{~K}$ and $300 \mathrm{~K}$ in magnetic fields up to $8 \mathrm{~T}$. Lakeshore controller allowed stabilizing temperature with the accu-

\footnotetext{
*corresponding author; e-mail: fedotov@bsu.by
}

racy of $\pm 0.005 \mathrm{~K}$ and enabled to use different sweeping rates of magnetic field and applied bias voltage.

\section{Results and discussion}

The concentration $N_{\mathrm{Sb}}$ corresponds to the average distance between impurity atoms of $6.2 \mathrm{~nm}$. Measurements of the resistivity $\rho$ in the absence of the external magnetic field revealed its strong temperature dependence at $T<25 \mathrm{~K}$; the changes in $\rho$ were more than one order of magnitude. Application of the magnetic field above 1.5 $\mathrm{T}$ showed a significant positive MR: it equals $50 \%$ for $B=8 \mathrm{~T}$ at $T=5 \mathrm{~K}$. In $B=2-8 \mathrm{~T}$ the $\mathrm{MR}$ is a squared function of $B$. It decreases with temperature practically vanishing above $T=25 \mathrm{~K}$, Fig. 1 . Therefore, two regions are distinguished from the viewpoint of MR: $\Delta T=5-25 \mathrm{~K}$, where the magnetoresistance is present, and $\Delta T=25-300 \mathrm{~K}$, where it is lack.

The characteristic feature of high temperature region, where the magnetoresistance is lack, is the presence of two activation energies, $\varepsilon_{1}=10.35 \mathrm{meV}$ (for the temperature range $\Delta T=90-300 \mathrm{~K}$ ) and $\varepsilon_{2}=1.725 \mathrm{meV}$ (for $\Delta T=25-90 \mathrm{~K})$, Fig. 2 .

Within the two-band model $\varepsilon_{1}$ represents the activation energy of an electron from the Fermi level in the lower-Hubbard band (LHB) to the conduction band, and $\varepsilon_{2}$ that of the extended states in the upper-Hubbard band (UHB). The UHB corresponds to the zone of states formed by the charged centers $\mathrm{D}^{-}$, while the LHB means the zone of states formed by the neutral centers $\mathrm{D}^{0}[3]$.

The performed analysis revealed that, for the case $B=0 \mathrm{~T}$ and $\Delta T=5-20 \mathrm{~K}$, the temperature dependence of the resistivity is linear in the $\ln \rho(0)-T^{-1 / 4}$ scale. Therefore, the model of low temperature VRH conductivity, for which low- $T$ resistivity is described by the Mott law, is applicable [1, 4], 


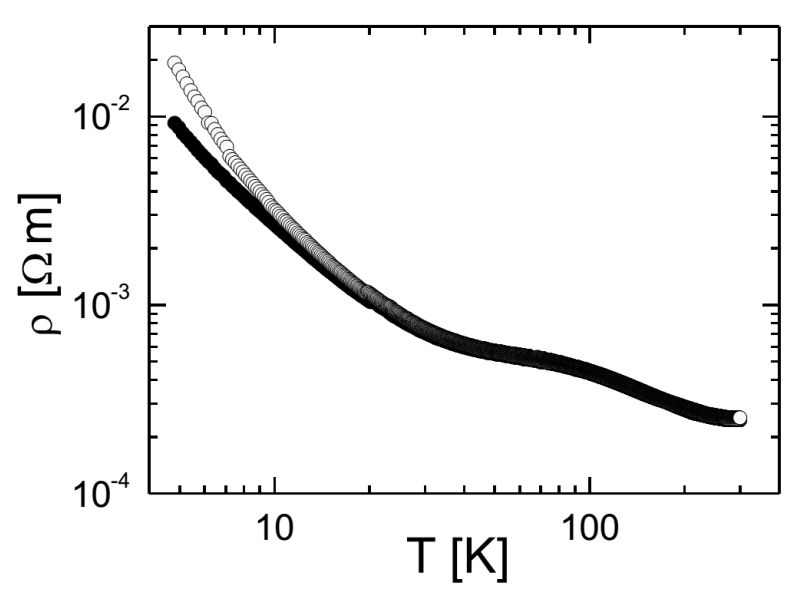

Fig. 1. Temperature dependence of the resistivity at $B=0$ (closed circles) and $B=8 \mathrm{~T}$ (open circles) for $\mathrm{Si}$ heavily doped with $\mathrm{Sb}$. The value of bias current is $I=10 \mu \mathrm{A}$

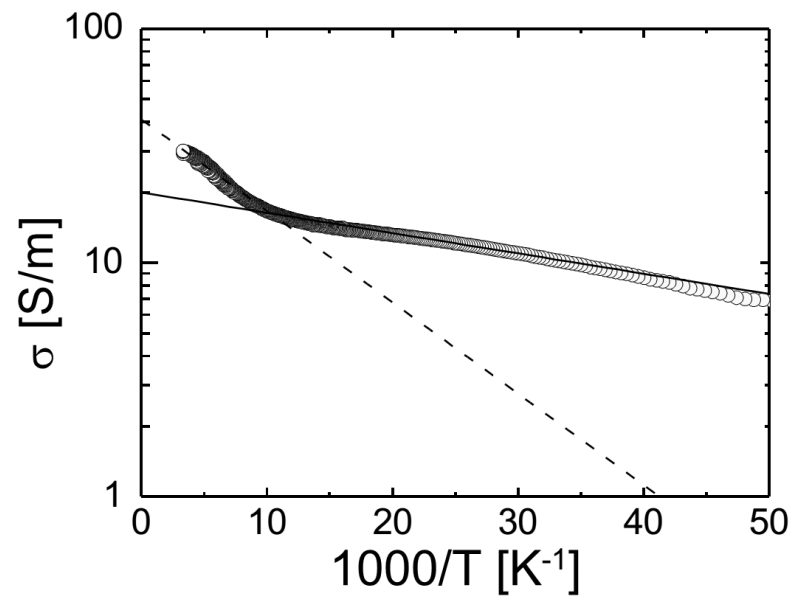

Fig. 2. Specific conductivity vs. $T^{-1}$ for the temperature range $\Delta T=25-300 \mathrm{~K}$. Experimental data (open circles) are approximated by two activation energies. Dashed line corresponds to the $\Delta T=90-300 \mathrm{~K}$, solid line is for $\Delta T=25-90 \mathrm{~K}$.

$$
\rho(T)=\rho_{0} \exp \left(\left(T_{0 \mathrm{M}} / T\right)^{1 / 4}\right),
$$

where $T_{0 \mathrm{M}}=\beta_{\mathrm{M}}\left[g\left(E_{\mathrm{F}}\right) a_{\mathrm{M}}^{3} k_{\mathrm{B}}\right]^{-1}$ is the Mott parameter, $\beta_{\mathrm{M}}=21.2 \pm 1.2$ is the constant, $g\left(E_{\mathrm{F}}\right)=$ const is density of states (DOS) of electron at the Fermi level, $k_{\mathrm{B}}$ - the Boltzmann constant, $a_{\mathrm{M}}$ - the localization radius of the states around the Fermi level, $\rho_{0}$ is the pre-exponential factor.

Estimations of the DOS and localization radius values have been obtained by plotting the experimental data in the Mott coordinates and application of valuation formulae without using the MR data $[5,6]$ :

$$
g\left(E_{\mathrm{F}}\right)=\frac{N_{\mathrm{d}}}{2 k_{\mathrm{B}}\left(T_{0 \mathrm{M}} T_{\mathrm{vM}}^{3}\right)^{1 / 4}},
$$

$$
a_{\mathrm{M}}=\frac{\beta_{\mathrm{M}}^{1 / 3}}{\left[k_{\mathrm{B}} T_{0 \mathrm{M}} g\left(E_{\mathrm{F}}\right)\right]^{1 / 3}},
$$

where $T_{\mathrm{vM}}$ is the temperature of the onset of the Mott VRH.

The results of calculations according to Eqs. (2), (3) are summarized in Table. The obtained within different approaches values of the Mott parameter and DOS are in good agreement with each other.

TABLE

Parameters of the VRH model obtained within different approaches.

\begin{tabular}{c|c|c}
\hline \hline $\begin{array}{c}\text { The method of } \\
\text { parameter estimation }\end{array}$ & $\begin{array}{c}\text { Equations } \\
(2),(3)\end{array}$ & $\begin{array}{c}\text { From the } \\
\text { MR data }\end{array}$ \\
\hline$T_{\mathrm{vM}}[\mathrm{K}]$ & $19-20$ & - \\
$T_{0 \mathrm{M}}[\mathrm{K}]$ & $1.524 \times 10^{4}$ & $1.524 \times 10^{4}$ \\
$a_{\mathrm{M}}[\mathrm{nm}]$ & 6.58 & 7.0 \\
$g\left(E_{\mathrm{F}}\right)\left[\mathrm{eV}^{-1} \mathrm{~cm}^{-3}\right]$ & $5.408 \times 10^{19}$ & $4.7 \times 10^{19}$
\end{tabular}

In Table parameters of the VRH model obtained from the MR measurements are also shown. The significant positive contribution to the MR of the form $\ln (\rho(H) / \rho(0)) \sim H^{2} f(T)$, which is usually related to the localized state wave function contraction in magnetic field, was observed. The joint analysis of $\rho(T)$ and $\operatorname{MR}(B)$ dependences leads to the possibility of finding of $g\left(E_{\mathrm{F}}\right)$ and $a_{\mathrm{M}}$ in self-consistent mode. Positive MR for the wave function contraction mechanism is expressed as $[4,7]$ :

$$
\ln \frac{\rho(H)}{\rho(0)}=t_{3} \frac{a_{\mathrm{M}}^{4}}{l_{H}^{4}}\left(\frac{T_{0 \mathrm{M}}}{T}\right)^{3 / 4},
$$

where $t_{3}=5 / 2016, l_{H}=\sqrt{\hbar c / q H}=\sqrt{\hbar / q B}$ is the magnetic length and $\rho(0)$ - the zero field resistivity.

On the base of Eq. (4) it is possible to obtain $a_{\mathrm{M}}$ values versus $B$ and $T$. These results are plotted in Fig. 3a,b. It follows from the analysis of Fig. 3 that, at $B>2 \mathrm{~T}$, the localization radius practically does not depend on $B$. At that some temperature dependence is present. In particular, for the temperature range $\Delta T=5-25 \mathrm{~K}$ the $a_{\mathrm{M}}$ values decrease from 8.5 to $\approx 6.0 \mathrm{~nm}$.

Within the obtained Mott parameters it is possible to evaluate the width of the energy band of the so-called essential states [4]:

$$
\varepsilon_{0}(T)=\left(k_{\mathrm{B}} T\right)^{3 / 4} /\left[g\left(E_{\mathrm{F}}\right) a_{\mathrm{M}}^{3}\right]^{1 / 4}=k_{\mathrm{B}} T\left(T_{0 \mathrm{M}} / T\right)^{1 / 4},
$$

which is in the range $\varepsilon_{0}=2.7-11 \mathrm{meV}$. This leads to the estimation of the DOS $N\left(\varepsilon_{0}\right)=2 g\left(E_{\mathrm{F}}\right) \varepsilon_{0}=$ $(2.7-9) \times 10^{17} \mathrm{~cm}^{-3}$ in this narrow band. This value agrees well with the real donor concentration. Moreover, the inequality $\varepsilon_{0}(T)>k_{\mathrm{B}} T$ is satisfied for $\Delta T=5-25 \mathrm{~K}$, i.e. the width of the narrow band is larger than the characteristic temperature energy $k_{\mathrm{B}} T$.

The most important result regarding estimations of localization radii from the MR data is related to the temperature dependence of $a_{\mathrm{M}}$. In this case the model of 

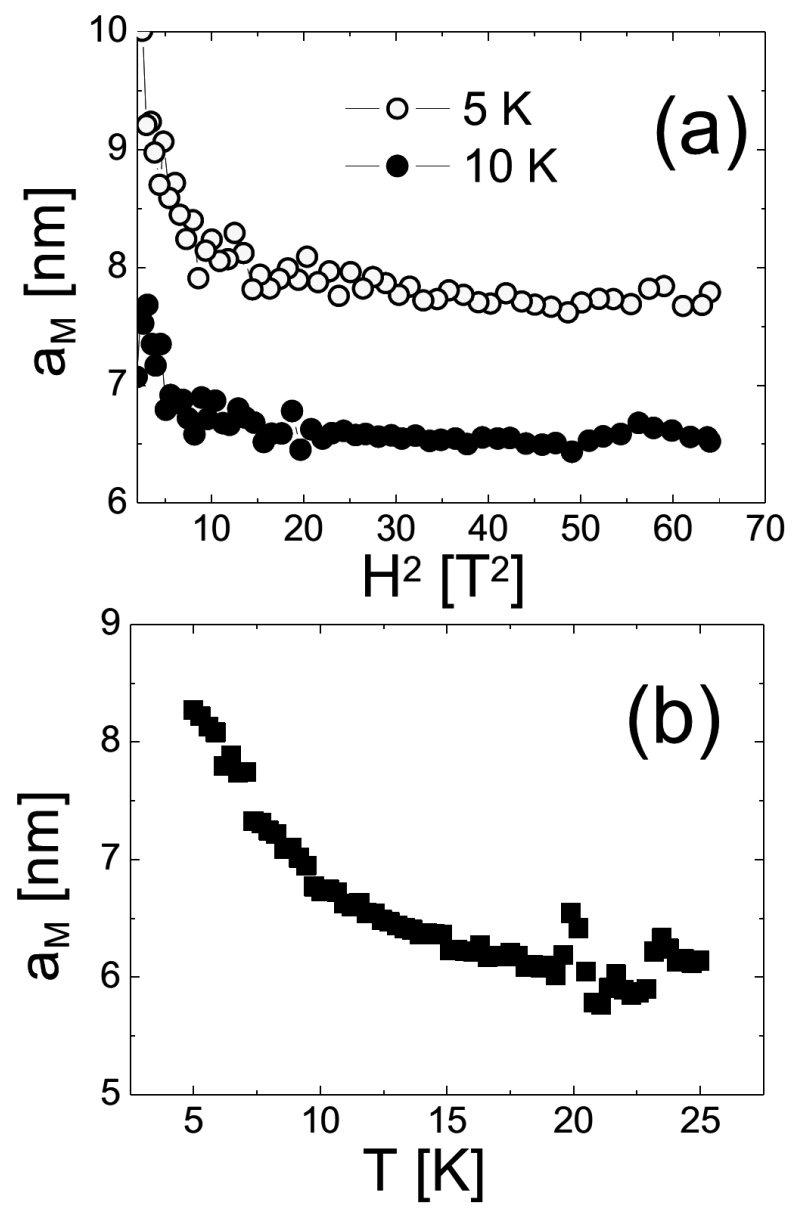

Fig. 3. Magnetic field dependences of $a_{\mathrm{M}}$ at two temperatures (a) and temperature dependence of $a_{\mathrm{M}}$ at $B=8 \mathrm{~T}(\mathrm{~b})$.

positive MR, which takes into account only the contraction of the wave function, is incomplete. To describe the MR adequately we need to take into account the spin polarized contribution, which reflects electron hopping not only by free localized states, but also by the occupied ones [8]. Typically, only intracenter correlations are considered and the long range column effects are neglected. In this case the resistance of the samples is determined by two types of tunnel transitions. To describe them in detail we assume that if $\mathrm{D}^{0}$ denotes singly occupied, or free state, and $\mathrm{D}^{-}$is for doubly occupied state, then $\mathrm{D}^{-}$ states should be of larger energy than $\mathrm{D}^{0}$ states. This difference is determined by the energy of repulsion between centers. But due to the dispersion of distances between centers energy levels $\mathrm{D}^{0}$ and $\mathrm{D}^{-}$will be overlapped. Therefore both $\mathrm{D}^{0}$ and $\mathrm{D}^{-}$states will be in the vicinity of the Fermi level.

So, the first type of hopping is realized only between $\mathrm{D}^{0}$ states, while the second one could occur between $\mathrm{D}^{-}$ and $\mathrm{D}^{-}, \mathrm{D}^{0}$ and $\mathrm{D}^{-}, \mathrm{D}^{-}$and $\mathrm{D}^{0}$ centers. All jumps of the second type include a double occupied center with oppo- site electron spins. Application of the external magnetic field polarizes these spin states, therefore the percentage of the second type transitions is reduced and magnetoresistance appears.

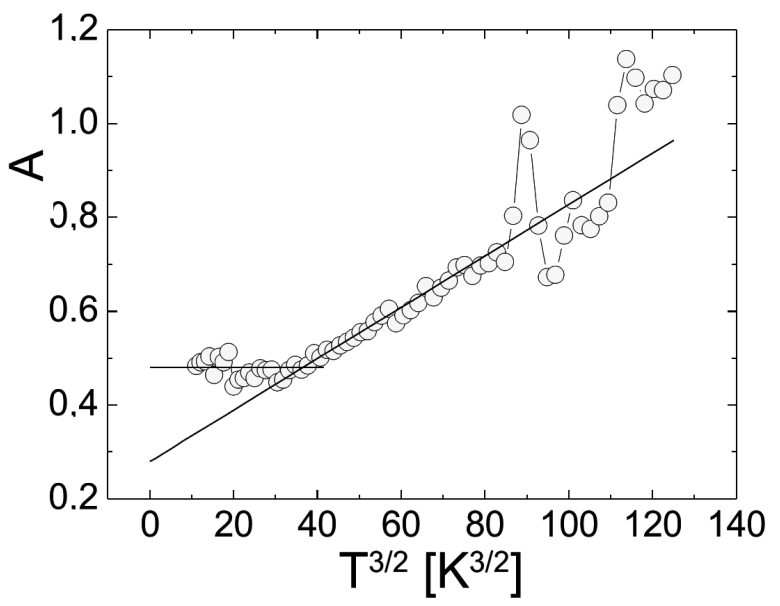

Fig. 4. Temperature dependence of the coefficient $A$ in Eq. (6) (points), showing two different mechanisms of hopping in heavily doped Si. For details see the text.

The first type jumps of electrons are characterized by the parameters $a_{1}$ and $g_{1}\left(E_{\mathrm{F}}\right)=g_{1}$, while the second type hopping depends on the parameters $a_{2}$ and $g_{2}\left(E_{\mathrm{F}}\right)=g_{2}$. The localization radius for states $\mathrm{D}^{-}$is larger than that of $\mathrm{D}^{0}$ state. At that the spatial part of the hopping integral is determined by the largest localization radius. Therefore, it is reasonably to assume that the parameter $a_{2}$ means the localization radius in $\mathrm{D}^{-}$ band. In this case the modified expression for MR is [8]:

$$
\begin{aligned}
& \ln \frac{\rho(H)}{\rho(0)}=\left(\frac{T_{0 \mathrm{M}}}{T}\right)^{1 / 4} \\
& \quad \times\left[\left(\frac{1}{1-A \tanh ^{2}\left(\mu_{\mathrm{B}} H / k_{\mathrm{B}} T\right)}\right)^{1 / 4}-1\right],
\end{aligned}
$$

where $A=\left(g_{2} a_{2}^{3}-g_{1} a_{1}^{3}\right) /\left(g_{2} a_{2}^{3}+g_{1} a_{1}^{3}\right)$. Here $g_{1}, g_{2}$ are DOS, $a_{1}, a_{2}$ are localization radii for two types of jumps, $T_{0 \mathrm{M}}=\frac{2 n_{\mathrm{c}}}{k_{\mathrm{B}}\left(g_{2} a_{2}^{3}+g_{1} a_{1}^{3}\right)}$, where $n_{\mathrm{c}}$ is a constant. The effect of the wave function contraction influences mainly jumps with the maximum length, i.e. the second type hopping. Consequently, influence of the wave function contraction on the MR could be accounted for by appying Eq. (6) assuming $a_{\mathrm{M}}=a_{2}$. Parameter $T_{0 \mathrm{M}}$ is known from the $\rho(T)$ measurements in zero field. By applying Eq. (6) for analysis of the experimental data regarding MR, it is possible to obtain values of the parameter $A$ at each temperature. Taking into account both the spin polarized mechanism and wave function contraction the temperature dependence of $A$ is expressed as [8]:

$$
A(T)=a_{\mathrm{S}}+b\left(T / T_{0 \mathrm{M}}\right)^{3 / 2},
$$

where constant $a_{\mathrm{S}}$ characterizes the contribution of spin polarized mechanism, and constant 


$$
b=4 t_{\mathrm{d}} a_{2}^{4}\left(q^{2} k_{\mathrm{B}}^{2} T_{0 \mathrm{M}}^{2} / \hbar^{2} \mu_{\mathrm{B}}^{2}\right)
$$

is related to the wave function contraction.

Analysis of the $A(T)$ dependence is present in Fig. 4. It is seen that, at low $T(\Delta T=5-11 \mathrm{~K})$, the coefficient $A$ is temperature independent which, according to Eq. (7), corresponds to the dominant spin polarized hopping. At $T>11 \mathrm{~K}$ mostly the wave function contraction determines hopping.

\section{Summary}

In conclusion, measurements of the resistivity versus temperature and magnetoresistance at different temperatures were performed for single crystalline silicon heavily doped with antimony. Range of temperature was changed between 5 and $300 \mathrm{~K}$, magnetic field was varied up to $8 \mathrm{~T}$.

It was established that, in the temperature range $\Delta T=25-300 \mathrm{~K}$, the resistivity is of activation type with two characteristic activation energies, $10.35 \mathrm{meV}$ and $1.725 \mathrm{meV}$, while in the low $T$ range, $\Delta T=5-25 \mathrm{~K}$, the VRH dominates. For the VRH the positive MR of $50 \%$ at $T=5 \mathrm{~K}$ was also observed. At temperatures above $25 \mathrm{~K}$ the MR effect vanishes.

Carried out according to various approaches evaluations of the model parameters showed that the value of the Mott parameter is $(1-1.5) \times 10^{4} \mathrm{~K}$, the localization radius is around $7-8 \mathrm{~nm}$ and the DOS at the Fermi level is in the range $(4.5-5.4) \times 10^{4} \mathrm{eV}^{-1} \mathrm{~cm}^{-3}$. The obtained on the base of MR measurements values of the localization radius revealed its temperature dependence, which led to the conclusion that it is insufficient to use only the Mott law in its classical form for MR and localization radius estimations. Within the models of either spin polarized hopping conductivity, or wave function contraction two temperature intervals were established, which reflects different contributions of these mechanisms. In the temperature range $\Delta T=5-11 \mathrm{~K}$ the spin dependent hopping dominates, while for $\Delta T=11-20 \mathrm{~K}$ both mechanisms should to be taken into account. This result is consistent with the general physical representation that with decreasing temperature the contribution of hopping by the occupied states increases.

\section{Acknowledgments}

The work was partially supported by the Belarusian State Scientific Program "Nanomaterials and Nanotechnologies", project \# 2.4.12.

\section{References}

[1] N.F. Mott, Metal-Insulator Transition, 2nd ed., Taylor and Francis, London 1990.

[2] P.P. Edwards, M.J. Sienko, Phys. Rev. B 17, 2575 (1978).

[3] A. Fujimoto, H. Kobori, T. Ohyama, S. Ishida, K. Satoh, T. Kusaka, Y. Kakehi, Physica B 324, 1 (2002)
[4] B.I. Shklovskii, A.L. Efros, Electronic Properties of Doped Semiconductors, Springer, Berlin 1984, p. 45.

[5] K.G. Lisunov, E.K. Arushanov, Ch. Kloc, U. Malang, E. Bucher, Phys. Status Solidi B 195, 227 (1996).

[6] K.G. Lisunov, E.K. Arushanov, G.A. Thomas, E. Bucher, J.H. Schön, J. Appl. Phys. 88, 4128 (2000).

[7] M.E. Raikh, J. Czingon, Q. Ye, F. Koch, W. Schoepe, K. Ploog, Phys. Rev. B 45, 6015 (1992).

[8] S.V. Demishev, A.A. Pronin, Phys. Solid State 48, 1363 (2006). 